\title{
Pain management within an enhanced recovery program after thoracic surgery
}

\author{
Calvin Thompson ${ }^{1}$, Daniel G. French ${ }^{2}$, Ioana Costache ${ }^{1}$ \\ ${ }^{1}$ Department of Anesthesiology and Pain Medicine, University of Ottawa, Ottawa, Ontario, Canada; ${ }^{2}$ Division Thoracic Surgery, Department of \\ Surgery, Dalhousie University, Halifax, Nova Scotia, Canada \\ Contributions: (I) Conception and design: All Authors; (II) Administrative support: None; (III) Provision of study materials or patients: None; (IV) \\ Collection and assembly of data: All Authors; (V) Data analysis and interpretation: None; (VI) Manuscript writing: All authors; (VII) Final approval \\ of manuscript: All authors. \\ Correspondence to: Calvin Thompson, MD, FRCPC. Assistant Professor, Department of Anesthesiology and Pain Medicine, The Ottawa Hospital, \\ University of Ottawa, 501 Smyth Road, Ottawa, ON, Canada. Email: cthompson@toh.ca.
}

\begin{abstract}
Evidence for ERAS within thoracic surgery (ERATS) is building. The key to enabling early recovery and ambulation is ensuring that postoperative pain is well controlled. Surgery on the chest is considered to be one of the most painful of surgical procedures for both open and minimally invasive surgery (MIS) approaches. Increasing use of MIS and improved perioperative care pathways has resulted in shorter length of stay (LOS), requiring patients to achieve optimal pain control earlier and meet discharge criteria sooner, sometimes on the same day as surgery. This requires optimizing pain control earlier in the postoperative recovery phase in order to enable ambulation and a better recovery profile, as well as to minimize the risk for development of chronic persistent postoperative pain (CPPP). This review will focus on the options for pain management protocols within an ERAS program for thoracic surgery patients (ERATS).
\end{abstract}

Keywords: Enhanced recovery after surgery (ERAS); thoracic surgery; multimodal; pain, analgesia

Submitted May 02, 2018. Accepted for publication Sep 21, 2018.

doi: $10.21037 /$ jtd.2018.09.112

View this article at: http://dx.doi.org/10.21037/jtd.2018.09.112

Enhanced recovery after surgery (ERAS) protocols have found widespread adoption for a variety of surgical procedures, and have been shown to improve patient outcomes $(1,2)$. Evidence for ERAS within thoracic surgery (ERATS) is building, but recommendations from the ERAS society are still pending (3). Minimally invasive surgery (MIS) procedures challenge our traditional analgesic regimens as they result in shorter length of stay (LOS), requiring patients to achieve optimal pain control earlier and meet discharge criteria sooner, sometimes on the same day as surgery. The key to enabling early recovery and ambulation is ensuring that postoperative pain is well controlled. Many elements of ERAS models of care have already been embraced in thoracic surgery, well before the wide usage of the term ERAS. These elements include aggressive pain control, fluid restriction, and early mobilization. Unfortunately, pioneering reports that described "fast tracking" of thoracic surgery patients only included a superficial mention of analgesia techniques and did not consider the importance of this element to reach their goal $(4,5)$. More recently, it has been suggested that two major barriers to fast tracking thoracic surgical patients are pain control and chest tube management (6). Surgery on the chest is considered to be one of the most painful of surgical procedures for both open and MIS approaches. In addition, poorly controlled postoperative pain can lead to development of chronic persistent postoperative pain (CPPP) (7). Our challenge is to optimize pain control earlier in the postoperative recovery phase in order to enable ambulation and a better recovery profile for these procedures, as well as to minimize the risk for development of CPPP (8). This review will focus on the options for 
pain management protocols within an ERAS program for thoracic surgery patients.

Attenuating the stress response to surgery is probably the most important element to enhancing recovery. Properly managed analgesia should achieve this goal, enabling a faster recovery (2). Traditional analgesic approaches for thoracic surgery have been reliant on opioids, physician driven, highly variable, and provider focused. ERAS approaches to analgesia are opioid sparing, increasingly standardized, evidence based, and patient focused (2). This model would aim to ensure the patient receives the best preoperative, intraoperative, postoperative, and discharge analgesics to ensure the best recovery profile. Adopting a standardized, collaborative, multidisciplinary, evidence-based approach will minimize practitioner variability, decrease those analgesic practices with higher associated side effects, improve flow of patient care, and improve patient functional recovery.

ERAS guidelines recommend the following multimodal pain management strategies (9):

(I) The use of a variety of analgesic medications to target different mechanisms of action in the peripheral and/or central nervous system;

(II) The use of regional anesthesia;

(III) Avoidance of opioids whenever possible;

(IV) Transitioning to oral medications as soon as possible.

Multimodal analgesia follows the concept that using several analgesic agents with different mechanisms of action may have synergistic effects in preventing and treating acute pain while reducing opioid related side effects (10). Suboptimal management of pain continues to be an issue. There are a number of pharmacologic and non-pharmacologic techniques available for optimizing multimodal analgesia (11). For thoracic surgery, consideration should be given to including acetaminophen, NSAIDs, NMDA receptor antagonists, anticonvulsants, beta blockers, alpha-2 agonists, glucocorticoids, opioids, central neuraxial techniques, surgical site infiltration, and regional anesthesia.

\section{Regional anesthesia}

Neural blockade with either central (epidural) or peripheral (paravertebral, intercostal, other blocks) are strongly advocated within any ERAS program for thoracic surgery. The use of properly placed local anesthetic greatly improves analgesia and decreases requirements for opioids. Catheter techniques have the benefit of prolonging analgesia to improve patient mobilization and recovery. A recent survey of thoracic anesthesiologists (members of the Canadian Anesthesiologists' Society) found analgesic techniques for VATS surgeries to be variable, as well as largely dictated by provider preferences (12). Epidural analgesia was preferred by $93 \%$ for open thoracotomy and $41 \%$ for VATS lobectomy. Only 14\% of respondents preferred paravertebral block for any VATS surgeries.

Epidurals may have a higher incidence of both urinary retention and hypotension than paravertebral blocks (13). Epidural infusions of combinations of local anesthetics and adjuvants targeting different pharmacologic pathways are more effective than a single agent infusion (Ex: bupivacaine $0.1 \%$ + fentanyl $2 \mathrm{mcg} / \mathrm{mL}$ + epinephrine $2 \mathrm{mcg} / \mathrm{mL}$ ) (14).

Most studies suggest that paravertebral blocks provide comparable analgesia to epidural infusion with greater hemodynamic stability, a better short-term side effect profile, and better preservation of pulmonary function (15). Many of these studies have the paravertebral catheters placed under direct vision by the surgeon. It is important for optimal function of the paravertebral catheter to be positioned with a standardized approach. The following describes our approach for a standardized, reproducible method of placing paravertebral catheters. Paravertebral catheters can be placed under direct vision through an open thoracotomy or VATS approach in 5 to 10 minutes. At the time of open thoracotomy, a narrow pocket in the plane between the chest wall and the parietal pleura is created using a blunt instrument such as a peanut or Kelly clamp. The pocket starts in the intercostal space at the posterolateral aspect of the thoracotomy incision and must extend to the sympathetic chain. It is important to avoid pleural puncture whereby leakage of local anesthetic can occur and possibly result in inadequate analgesia. A narrow pocket is better than a wide pocket in order to ensure less dispersion of the infused anesthetic and optimize the amount of local anesthetic entering the paravertebral space. A large bore needle is used to pass the catheter through the chest wall, and the proximal end of the catheter is placed in the pocket that has been created, with the tip advanced to the sympathetic chain.

For placement during a VATS technique, a small hole is made in the parietal pleura $1 \mathrm{~cm}$ lateral to the sympathetic chain. A blunt instrument (such as a VATS DeBakey) is then used to create a narrow track between the parietal pleura and chest wall three to four rib spaces superior to the entry point in the pleura. It is important to avoid puncture 
of the pleura while crossing the ribs and to keep the track as narrow as possible. The catheter is passed through the chest wall in a similar manner to the open technique. The catheter is advanced to the most superior aspect of the track.

As the chest is being closed, the paravertebral catheter is usually bolused with $20 \mathrm{~mL}$ of bupivacaine $0.25 \%$ in aliquots of $2-3 \mathrm{~mL}$ every $2-3$ minutes. The literature reports variable local anesthetic concentrations for continuous infusions ranging from bupivacaine $0.1-0.25 \%$ at $5-12 \mathrm{~mL} / \mathrm{h}$ to ropivacaine $0.2 \%$ at $4 \mathrm{~mL} / \mathrm{h}$ (16). Higher doses such as ropivacaine $0.375 \%$ at $12 \mathrm{~mL} / \mathrm{hr}$ can safely be infused. Patients are transitioned to oral pain medications and discharged 12 to 24 hours after removal of the catheter.

Intercostal blocks have the appeal of ease of administration, and can be administered as a single injection or continuous infusion through placement of a catheter. Direct infiltration under direct vision ensures correct dispersion of local anesthetic versus blind intercostal blocks.

Continuous catheter techniques for VATS/thoracotomy have the benefit of ongoing titrated analgesia. These require extra time and expertise for catheter placement, as well as ongoing monitoring. For short LOS patients, a continuous intercostal or wound infiltration catheter may be used with an ambulatory pump once the patient is discharged. Long acting local anesthetics are available in some countries (e.g., liposomal bupivacaine) and have shown a benefit of prolonged analgesia for intercostal nerve block, removing the need for catheter techniques (17).

The addition of dexamethasone to perineural local anesthetic solution has consistently been shown to prolong the duration of analgesia for short, medium, and long acting local anesthetics (18). Dose finding studies are currently underway, but a dose of $4 \mathrm{mg}$ seems efficacious without any reported adverse effects. Clinicians must be aware that perineural dexamethasone represents an off-label use, and solutions free of preservatives should be used. It is uncertain if this effect of dexamethasone is due to systemic effects as it has been shown in some studies to be equally achieved with IV administration (18). The addition of dexamethasone IV $(8 \mathrm{mg})$ has been shown to reduce postoperative pain, as well as to decrease opioid requirements and side effects such as postoperative nausea and vomiting (19).

Over the last several years, new techniques to block the chest wall have been described. These include the serratus plane (20,21), retrolaminar (22), intercostal/paraspinal (23), rhomboid/intercostal (24), erector spinae plane (ESP) (25), and mid-point transverse process to pleura (MTP) (26) blocks. Of these, the intercostal/paraspinal block has been described for thoracic surgery (23), the serratus plane block has been described for thoracotomy analgesia (20) and rib fractures (27), and ESP block described for thoracic pain $(25,28)$, rib fractures (29), post thoracotomy pain syndrome (30), and a number of other indications.

It is important to note that all of these descriptions have been in case reports only, with no formal trials to show efficacy. As all of these blocks are targeting either the nerve roots in the paravertebral space, or the lateral branch of the intercostal nerves more laterally, they may prove to be of benefit for thoracic surgery because of their ease of insertion and lack of side effects or complications associated with epidural or paravertebral blockade

\section{Patient preparation}

Pain related to surgery is one of the most commonly asked patient questions. Patient education is an essential components of ERAS programs, and this plan should include a review of anticipated discomfort associated with the planned surgery, as well as all options to minimize pain in order to enable best recovery. Managing a patient's expectations around pain control will greatly assist with the recovery process. Patients with preoperative pain should ensure they continue taking their analgesics perioperatively.

\section{Acetaminophen}

Acetaminophen is a building block of analgesia and recommended within a stepwise approach to pain management. It has a very favourable safety profile, and should be routinely used for mild to moderate pain as part of a multimodal regimen. The use of oral versus rectal acetaminophen is preferred due to the variability in rectal absorption. Intravenous (IV) acetaminophen may have advantages given the reliable pharmacokinetics and ease of administration, but its use may be limited by a potentially higher cost as well as availability.

\section{Non-steroidal anti-inflammatory drugs (NSAIDs)}

NSAIDs have been shown to reduce opioid consumption and opioid related side effects when used in multimodal analgesic programs $(15,19)$. NSAIDs have been promoted within standardized approaches to analgesia for thoracotomy (31). Selective NSAIDs [cyclooxygenase-2 (COX-2) inhibitors] may be preferred when compared 
to non-selective NSAIDs as they have a more favourable adverse effect profile with regards to surgical bleeding, gastrointestinal ulceration, and renal dysfunction. It remains to be determined which specific NSAID, dose, or method of administration offers any benefit over another.

\section{Gabapentinoids}

Procedures on the thorax carry significant neuropathic sequelae such as allodynia, burning sensation, and dysesthesia, in large part attributed to trauma to intercostal nerves. This can result in CPPP (15). The anticonvulsants gabapentin and pregabalin are gamma-aminobutyric acid (GABA) analogues that target neuropathic pain pathways. These have been shown to reduce postoperative opioid requirements and decrease acute and chronic pain when added to a multimodal analgesia regimen (19). Pregabalin has better bioavailability and reaches therapeutic levels more rapidly than Gabapentin. Variable dosing of gabapentin and pregabalin are included in analgesia protocols [e.g., Gabapentin $300 \mathrm{mg}$ po TID $\times 30$ days (32), Pregabalin 50 $\mathrm{mg}$ po TID]. These medications can have limiting side effects which include reversible blurred vision, sedation, somnolence, and dizziness. It is difficult to determine a "one size fits all" for standardized protocols. Reduced dosing should be considered for patients with renal dysfunction, those with increased risk for falls, or those experiencing dizziness. Our experience suggests a benefit with pregabalin $25 \mathrm{mg}$ po $\mathrm{q} 8 \mathrm{~h}$ as an adjunct, which is a lower dose than previously reported. Perioperative administration of pregabalin has been shown to be effective in reducing pain scores and opioid consumption for thoracotomy patients (33). A recent review of pregabalin suggests restricting its use to procedures associated with pronociceptive mechanisms, with thoracic surgery being a good example. The magnitude of benefit however might be less than previously suggested (34). The optimal dosing, timing, and duration of administration of these medications, as well as appropriate patient selection (i.e., renal dysfunction, the elderly patient with fall risk) remains to be determined.

\section{Narcotics}

Opioids remain an essential component of any analgesic protocol, but adverse effects such as sedation, postoperative nausea and vomiting, urinary retention, ileus, and respiratory depression may delay discharge. ERAS goals to minimize opioid use should be kept in mind when developing ERATS programs. Opioids should be used as rescue analgesics for poorly controlled analgesia when multi-modal non-opioid medications are inadequate for pain control. Transition to the early use of oral opioids is also preferable within an ERAS program in order to avoid reliance on IV medications and move towards readiness for discharge. Internationally, many centers have abandoned the routine use of IV PCA. A Canadian survey suggests that $27 \%$ of anesthesiologists favour IV PCA use for VATS lobectomies, while $46 \%$ favour it for VATS minor resections (12). Tramadol is a weak opiate receptor agonist with an improved side effect profile compared to other opioids and has been recommended within ERAS programs for moderate to severe pain before progression to stronger opioids for rescue analgesia.

Several authors have published their experience with protocols, but many are based on local experience rather than clinical evidence $(32,35)$. With varying access to medications internationally, and without controlled outcome measurements, it is difficult to draw recommendations from these publications. It is a challenge to suggest a "one size fits all" approach when different patient considerations need to be considered (age, fitness/frailty, renal function, and comorbidities).

\section{Ketamine}

Ketamine is an N-methyl-D-aspartate (NMDA) receptor antagonist that reduces postoperative opioid requirements (19). Low dose ketamine added to IV PCA morphine has been shown to decrease opioid requirements with lower pain scores post thoracotomy (36). Ketamine has not been shown to decrease chronic post thoracotomy pain (37). Ketamine is appealing as it is not associated with respiratory depression, and the addition of low dose ketamine should be considered as part of the intraoperative analgesia plan within an ERATS model.

\section{Other}

There are emerging techniques to minimize opioid administration, but there is insufficient experience to recommend these within an ERATS program. Alpha2 agonists (clonidine and dexmedetomidine) have shown promise within multimodal analgesia regimens. Dexmedetomidine administered perioperatively for thoracotomy patients has shown decreased opioid consumption with adequate analgesia, but requires 
increased monitoring because of the risk of hypotension (38). Increased sedation with these medications might limit their use within an ERATS program.

Esmolol infusions have been shown to reduce both intraoperative and postoperative opioid consumption, and have the additional advantage of blunting cardiovascular responses, possibly reducing adverse cardiac events $(19,39)$.

The surgical technique for thoracotomy and VATS may have impact on the pain and recovery profile. There is growing evidence to show that techniques offering some form of protection to the intercostal nerve are associated with reduced postoperative pain and analgesic consumption (15).

It has been suggested that single port VATS lobectomy may decrease nerve injury and reduce post operative pain (40).

\section{ERAS for esophagectomy}

There are currently no ERAS guidelines for esophagectomy, but standardized pathways have shown improved outcomes (41). Esophagectomy remains a very high-risk procedure and patients would likely benefit from ERAS models of care. Recent studies suggest ERAS for esophagectomy seems favourable, logical and safe $(42,43)$. Optimal analgesia techniques have yet to be determined. Similar to initial papers on ERAS for pulmonary resection, the details of analgesia to optimize recovery for esophagectomy are limited. Analgesia for esophagectomy remains controversial for several reasons, primarily because approaches to esophageal resection are variable (open, hybrid, MIE). Epidurals remain gold standard with documented benefits: improved postoperative pain relief, earlier recovery of gastrointestinal function, earlier extubation, and earlier mobilization (44). Additional potential benefits of epidural analgesia include: reduced pulmonary complications (45), reduced anastomotic leak (46), and improved gastric conduit microcirculation (47). Vigilance with epidural management is important because of the risk of hypotension and concern of increased risk for anastomotic leak. Differences in surgical preference for the initiation of oral intake, placement of a jejunostomy tube, and availability of liquid medications can affect types of oral analgesics options early in recovery. IV PCA narcotics are frequently used because of limitations of drug delivery. ERAS goals of limiting opioids and transitioning from intravenous to oral medications are often delayed in esophagectomy patients. NSAID use for esophagectomy patients has to be used cautiously. Normal renal function postoperatively should be documented before considering NSAIDs. NSAIDs have been associated with a possible increase in anastomotic leaks for colorectal surgery (48). It is uncertain if there are similar concerns related to anastomotic leak for foregut/esophageal resection procedures.

The paravertebral space is continuous between the thorax and abdomen. Paravertebral catheters have been effective for inguinal hernia repair (49), Paravertebral catheters may provide regional anaesthesia for both the chest and abdominal incisions in esophagectomy but remains an area for future study.

\section{Conclusions}

ERAS models of care are here to stay. We anticipate the term "ERAS Pathway" will likely fade for the reason that ERAS elements are simply a step away from variation in practice towards standardization of best care practices. Optimal analgesia has always been a focus for thoracic surgery patients because of significant pain associated with these procedures and recognition that poor recovery is associated with inadequate pain control. The challenge for successful implementation of a standardized analgesic plan requires engagement from all care providers. It has been demonstrated that increased compliance with an ERAS pathway is associated with improved clinical outcomes after resection for primary lung cancer (50). Patients need to follow analgesic instructions and participate in providing feedback on adequacy of pain control and side effects. Preoperatively, staff need to educate patients and help set patient expectations around the analgesic plan. Anesthesiologists should avoid long acting sedatives, use multimodal pain management and opioid sparing techniques with regional anesthesia wherever possible, and maintain communication with the team when following patients on an Acute Pain Service. Surgeons should continue opioid sparing multimodal pain management postoperatively and upon discharge. Nurses should continue to educate patients postoperatively with regards to best analgesic care plans.

Inclusion of a standardized multimodal analgesia plan is an essential component to an ERATS program. Table 1 presents a summary of analgesia to consider within an ERATS program. The relative influence and importance of any individual analgesic component is difficult to determine, and depends on the individual patient characteristics as well as availability of certain modalities. Ensuring compliance with a specific analgesic protocol within a chosen institutional ERAS program is suggested as the most important element for improved clinical outcomes. 
Table 1 Enhanced analgesia pathway for thoracic surgery

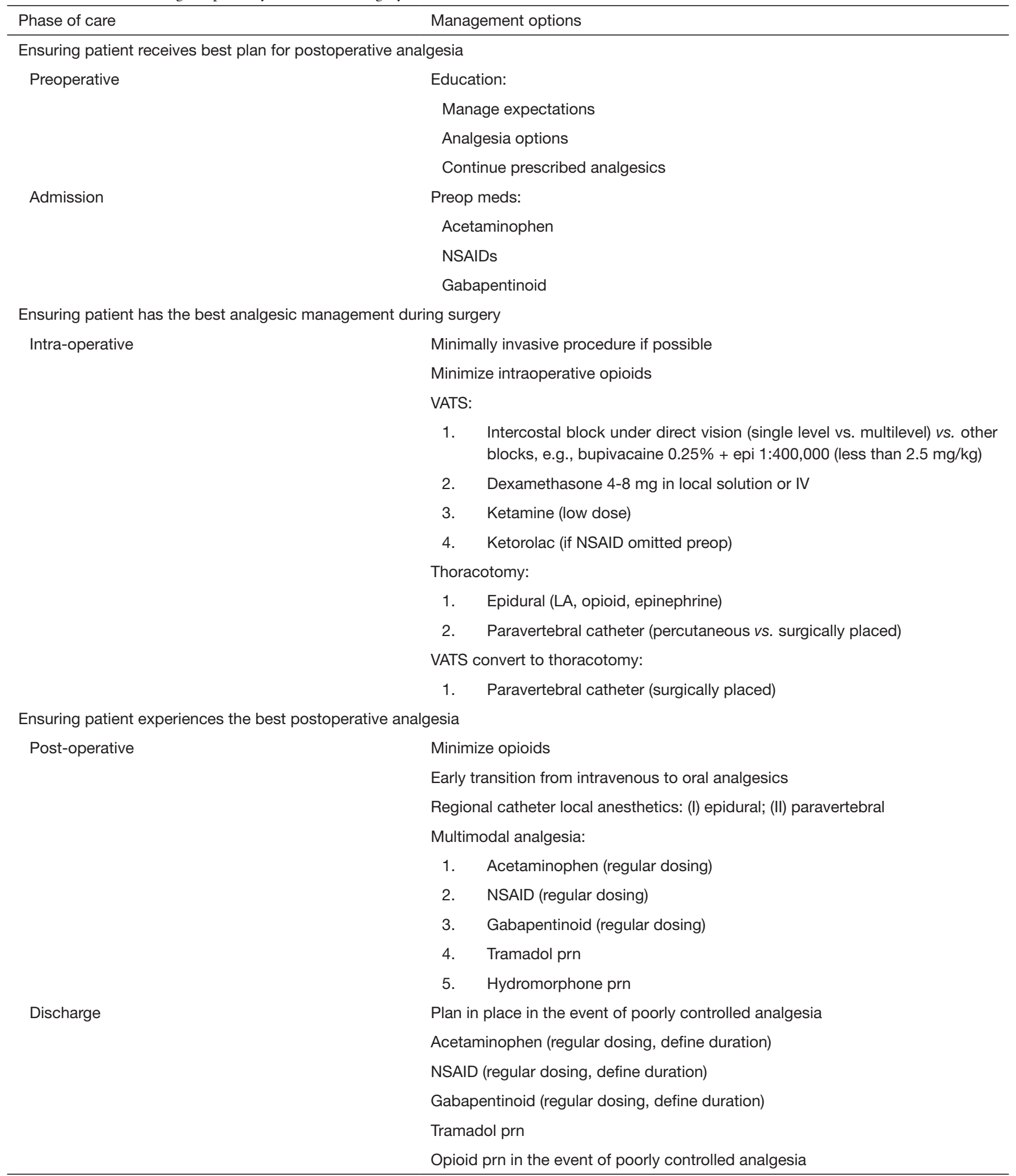
Adapted from NHS "Enhanced Recovery Care Pathways". VATS, video-assisted thoracic surgery; NSAID, non-steroidal anti-inflammatory drugs. 


\section{Acknowledgements}

None.

\section{Footnote}

Conflicts of Interest: The authors have no conflicts of interest to declare.

\section{References}

1. Ljungqvist, O. Scott, M. Fearon, K.C. Enhanced recovery after surgery: A Review. JAMA Surg 2017;152:292-8.

2. Carli F. Physiologic considerations of Enhanced Recovery After Surgery (ERAS) programs: implications of the stress response. Can J Anaesth 2015;62:110-9.

3. ERAS Society. Available online: http://erassociety.org/

4. Cerfolio RJ, Pickens A, Bass C, et al. Fast Tracking Pulmonary Resection. J Thorac Cardiovasc Surg 2001;122:318-24.

5. McKenna RJ, Mahtabifard A, Pickens A, et al. FastTracking After Video-Assisted Thoracoscopic Surgery Lobectomy, Segmentectomy, and Pneumonectomy. Ann Thorac Surg 2007;84:1663-7; discussion 1667-8.

6. Brown LM. "Moving right along" after lung resection, but the data suggest "not so fast". J Thorac Cardiovasc Surg 2016;151:715-6.

7. Kehlet H, Jensen TS, Woolf CJ. Persistent postsurgical pain: risk factors and prevention. Lancet 2006;367:1618-25.

8. Gerner P. Postthoracotomy Pain management problems. Anesthesiol Clin 2008;26:355-67.

9. Chou R, Gordon DB, de Leon-Casasola OA, et al. Management of Postoperative Pain: A Clinical Practice Guideline from the American Pain Society, the American Society of Regional Anesthesia and Pain Medicine, and the American Society of Anesthesiologists Committee on Regional Anesthesia, Executive Committee and Administrative Council. J Pain 2016;17:131-57.

10. Young A, Buvanendran A. Recent advances in multimodal analgesia. Anesthesiol Clin 2012;30:91-100.

11. Kirksey MA, Haskins SC, Cheng J, et al. Local Anesthetic Peripheral Nerve Block Adjuvants for Prolongation of Analgesia: A Systematic Qualitative Review. PLoS One 2015;10:e0137312.

12. Shanthanna H, Moisuik P, O'Hare T, et al. Survey of Postoperative Regional Analgesia for Thoracoscopic Surgeries in Canada. J Cardiothorac Vasc Anesth. 2018. [Epub ahead of print].
13. Júnior Ade P, Erdmann TR, Santos TV, et al. Comparison between continuous thoracic epidural and paravertebral blocks for postoperative analgesia in patients undergoing thoracotomy: Systematic review. Braz J Anesthesiol 2013;63:433-42.

14. Niemi G, Breivik H. Adrenaline markedly improves thoracic epidural analgesia produced by low-dose infusion bupivacaine, fentanyl and adrenaline after major surgery. A randomized, double-blind, cross over study with and without adrenaline. Acta Anaesthesiol Scand 1998;42:897-909.

15. Maxwell C, Nicoara A. New developments in the treatment of acute pain after thoracic surgery. Curr Opin Anaesthesiol 2014;27:6-11.

16. Stoelting RK, Hillier SC. Pharmacology and Physiology in Anesthetic Practice. 4th edition. Lippincott Williams \& Wilkins, 2012.

17. Khalil KG, Boutrous ML, Irani AD, et al. Operative Intercostal Nerve Blocks with Long -Acting Bupivacaine Liposome for Pain Control After Thoracotomy. Ann Thorac Surg 2015;100:2013-8.

18. Albrecht E, Kern C, Kirkham KR. A systematic review and meta-analysis of perineural dexamethasone for peripheral nerve blocks. Anaesthesia 2015;70:71-83.

19. Tan M, Law LS, Gan TJ. Optimizing pain management to facilitate Enhanced Recovery After Surgery pathways. Can J Anaesth 2015;62:203-18.

20. Okmen K, Okmen B, Uysal S. Serratus anterior plane (SAP) block used for thoracotomy analgesia: a case report. Korean J Pain 2016;29:189-92.

21. Blanco R, Parras T, McDonnell JG, et al. Serratus plane block: a novel ultrasound-guided thoracic wall nerve block. Anaesthesia 2013;68:1107-13.

22. Murouchi T, Yamakage M. Retrolaminar block: analgesic efficacy and safety evaluation. J Anesth 2016;30:1003-7.

23. Roué C, Wallaert $M$, Kacha $M$, et al. Intercostal/ paraspinal nerve block for thoracic surgery. Anaesthesia 2016;71:112-3.

24. Elsharkawy H, Saifullah T, Kolli S, et al. Rhomboid intercostal block. Anaesthesia 2016;71:856-7.

25. Forero M, Adhikary SD, Lopez H, et al. The erector spinae plane block: a novel analgesic technique in thoracic neuropathic pain. Reg Anesth Pain Med 2016;41:621-7.

26. Costache I, de Neumann L, Ramnanan CJ, et al. The midpoint transverse process to pleura (MTP) block: A new endpoint for thoracic paravertebral block. Anaesthesia 2017;72:1230-6.

27. Durant E, Mantuani D. Ultrasound-guided serratus plane block for ED rib fracture pain control. Am J Emerg Med 
2017;35:197.e3-197.e6.

28. Chin KJ, Adhikary S, Sarwani N, et al. The analgesic efficacy of pre-operative bilateral erector spinae plane (ESP) blocks in patients having ventral hernia repair. Anaesthesia 2017;72:452-60.

29. Hamilton DL, Manickam B. Erector spinae plane block for pain relief in rib fractures. Br J Anaesth 2017;118:474-5.

30. Forero M, Rajarathinam M, Adhikary S, et al. Erector spinae plane (ESP) block in the management of post thoracotomy pain syndrome: a case series. Scand J Pain 2017;17:325-9.

31. Procedure specific postoperative pain management. Thoracotomy 2011. Available online: http://postoppain.org

32. Mehran RJ, Martin LW, Baker CM, et al. Pain management in an Enhanced Recovery Pathway After Thoracic Surgical Procedures. Ann Thorac Surg 2016;102:e595-6.

33. Sidiropoulou T, Giavasopoulos E, Kosotopanagiotou G, et al. Perioperative Pregabalin for Postoperative Pain Relief after Thoracotomy. J Anesth Surg 2016;3:1-6.

34. Eipe N, Penning J, Yazdi F, et al. Perioperative use of pregabalin for acute pain - a systematic review and metaanalysis. Pain 2015;156:1284-300.

35. Zvara DA. Enhanced Recovery for thoracic surgery. Conferencias Magistrales 2017;40:S340-4.

36. Mathews TJ, Churchhouse AMD, Housden T, et al. Does adding ketamine to morphine patient controlled analgesia safely improve post-thoracotomy pain? Interact Cardiovasc Thorac Surg 2012;14:194-9.

37. Mendola C, Cammarota G, Netto R, et al. S(+) -ketamine for control of perioperative pain and prevention of post thoracotomy pain syndrome: a randomized, double blind study. Minerva Anestesiologica 2012;78:757-66.

38. Ramsay MA, Newman KB, Leeper B, et al.

Dexmedetomidine Infusion for Analgesia up to 48 hours after Lung Surgery Performed by Lateral Thoracotomy. Proc (Bayl Univ Med Cent) 2014;27:3-10.

39. Gelineau AM, King MR, Ladha KS, et al. Intraoperative Esmolol as an Adjunct for Postoperative Opioid and Postoperative Pain Reduction: A Systematic Review, Meta-analysis, and Meta-regression. Anesth Analg 2018;126:1035-49.

40. French DG, Thompson C, Gilbert S. Transition from multiple port to single port video-assisted thoracoscopic anatomic pulmonary resection: early experience and comparison of perioperative outcomes. Ann Cardiothorac Surg 2016;5:92-9.

41. Low DE, Kunz S, Schembre D, et al. Esophagectomy - It's not just about mortality anymore: Standardized perioperative clinical pathways improve outcomes in patients with esophageal cancer. J Gastrointest Surg 2007;11:1395-402.

42. Findlay JM, Gillies RS, Millo J, et al. Enhanced Recovery for Esophagectomy: A Systematic Review and Evidence Based Guidelines. Ann Surg 2014;259:413-31.

43. Giacopuzzi S, Weindelmayer J, Treppiedie E, et al. Enhanced Recovery after surgery protocol in patients undergoing esophagectomy: a single center experience. Dis Esophagus 2017;30:1-6.

44. Neal JM, Wilcox RT, Allen HW, et al. Near-total esophagectomy: the influence of standardized multimodal management and intraoperative fluid restriction. Reg Anesth Pain Med 2003;28:328-34.

45. Zingg U, Smithers BM, Gotley DC, et al. Factors associated with postoperative pulmonary morbidity after esophagectomy for cancer. Ann Surg Oncol 2011;18:1460-8.

46. Michelet P, D'Journo XB, Roch A, et al. Perioperative Risk Factors for Anastomotic Leakage after Esophagectomy. Chest 2005;128:3461-6.

47. Lázár G, Kaszaski J, Abraham S. Thoracic epidural anesthesia improves the gastric microcirculation during experimental gastric tube formation. Surgery 2003;134:799-805.

48. Bhangu A, Singh P, Fitzgerald EF, et al. Postoperative Nonsteroidal Anti-inflammatory Drugs and Risk of Anastomotic Leak: Meta-Analysis of Clinical and Experimental Studies. World J Surg 2014;38:2247-57.

49. Klein SM, Greengrass RA, Weltz C, et al. Paravertebral somatic nerve block for outpatient inguinal herniorrhaphy: An expanded case report of 22 patients. Reg Anesth Pain Med 1998;23:306-10.

50. Rogers LJ, Bleetman D, Messenger DE, et al. The impact of enhanced recovery after surgery (ERAS) protocol compliance on morbidity from resection for primary lung cancer. J Thorac Cardiovasc Surg 2018;155:1843-52.
Cite this article as: Thompson C, French DG, Costache I. Pain management within an enhanced recovery program after thoracic surgery. J Thorac Dis 2018;10(Suppl 32):S3773-S3780. doi: $10.21037 /$ jtd.2018.09.112 